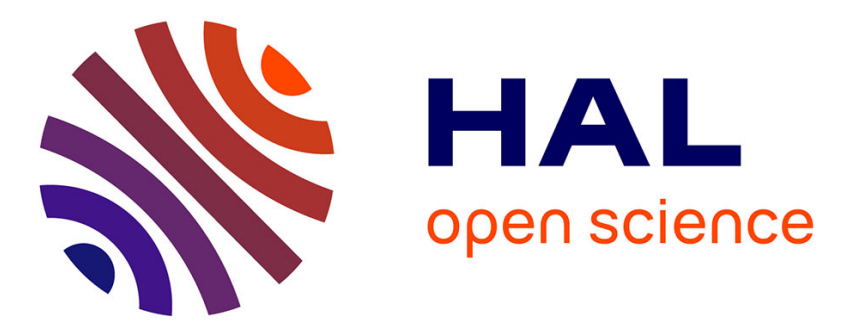

\title{
NFV Orchestration Platform for 5G over On-the-fly Provisioned Infrastructure
}

Nazih Salhab, Rana Rahim, Rami Langar

\section{To cite this version:}

Nazih Salhab, Rana Rahim, Rami Langar. NFV Orchestration Platform for 5G over On-the-fly Provisioned Infrastructure. IEEE INFOCOM 2019 - IEEE Conference on Computer Communications Workshops (INFOCOM WKSHPS), Apr 2019, Paris, France. pp.971-972, 10.1109/INFCOMW.2019.8845141 . hal-02566934

\section{HAL Id: hal-02566934 https://hal.science/hal-02566934}

Submitted on 29 May 2020

HAL is a multi-disciplinary open access archive for the deposit and dissemination of scientific research documents, whether they are published or not. The documents may come from teaching and research institutions in France or abroad, or from public or private research centers.
L'archive ouverte pluridisciplinaire HAL, est destinée au dépôt et à la diffusion de documents scientifiques de niveau recherche, publiés ou non, émanant des établissements d'enseignement et de recherche français ou étrangers, des laboratoires publics ou privés. 
THIS IS AN AUTHOR-CREATED POSTPRINT VERSION, A.K.A. ACCEPTED VERSION, AND NOT THE PUBLISHED VERSION AS IT MIGHT BE DOWNLOADED FROM IEEE XPLORE, TAKING INTO CONSIDERATION REVIEWERS COMMENTS.

Below are some Frequently Asked Questions (FAQs) (Excerpt from IEEE Author FAQ):

https://www.ieee.org/content/dam/ieee-org/ieee/web/org/pubs/author_faq.pdf

\section{Originality of Content}

- Does IEEE consider an author posting her paper on preprint servers or on her company's web sites to be a form of prior publication, which may then disqualify the paper from further editorial consideration?

No. IEEE policy allows an author to submit previously posted papers to IEEE publications for consideration as long as she is able to transfer copyright to IEEE, i.e., she had not transferred copyright to another party prior to submission.

\section{Authors' Rights to Post Accepted Versions of Papers}

- Can an author post his IEEE copyrighted paper on his personal or institutions' servers? Yes. An author is permitted to post his IEEE copyrighted paper on his personal site and his institution's server, but only the accepted version of his paper, not the published version as might be downloaded from IEEE Xplore.

- Can an author post his manuscript on a preprint server such as TechRxiv or ArXiv? Yes. The IEEE recognizes that many authors share their unpublished manuscripts on public sites. Once manuscripts have been accepted for publication by IEEE, an author is required to post an IEEE copyright notice on his preprint. Upon publication, the author must replace the preprint with either 1 ) the full citation to the IEEE work with Digital Object Identifiers (DOI) or a link to the paper's abstract in IEEE Xplore, or 2) the accepted version only (not the IEEEpublished version), including the IEEE copyright notice and full citation, with a link to the final, published paper in IEEE Xplore.

Disclaimer:

This work was accepted for publication in the IEEE. Final version after revision is accessible through: https://ieeexplore.ieee.org/Xplore/home.jsp

\section{IEEE Xplore}

Copyright:

CIEEE. Personal use of this material is permitted. Permission from IEEE must be obtained for all other uses, in any current or future media, including reprinting/republishing this material for advertising or promotional purposes, creating new collective works, for resale or redistribution to servers or lists, or reuse of any copyrighted component of this work in other works. 


\section{NFV Orchestration Application for 5G over On-the-fly Provisioned Infrastructure}

\author{
Nazih SALHAB \\ LIGM, U-PEM, University Paris-Est, France \\ LTRM, EDST, Lebanese University \\ Paris, France and Tripoli, Lebanon \\ nazih.salhab@u-pem.fr
}

\author{
Rana RAHIM \\ LTRM-EDST \\ Faculty of Science \\ Lebanese University \\ rana.rahim@ul.edu.lb
}

\author{
Rami LANGAR \\ LIGM-CNRS UMR 8049 \\ $U$-PEM, F-77420 \\ Marne-la-Vallée, Paris, France \\ rami.langar@u-pem.fr
}

\begin{abstract}
Multiple services with heterogeneous characteristics are anticipated with fifth generation of mobile communications (5G). Each requires an infrastructure tuned to serve requirements. To maintain affordability, operators will provide these services over a common infrastructure. We talk about provisioning Network Slices that are tailored to demands. A slice is a logical network built using Virtualized Network Functions (VNFs) that are enabled by leveraging Network Function Virtualization (NFV) and Software-Defined Networking (SDN). Orchestration is key for saving costs when tailoring infrastructures due to scarcity of resources. It enables operators to supply infrastructure with resources, meeting exactly with demands.

In this demo, we present an on-the-fly provisioned cluster used to provision $5 \mathrm{G}$ Core Network $(\mathrm{CN})$ as a Service $(\mathrm{CNaaS})$. Our platform is designed for high-availability with self-healing feature. It uses Docker Swarm as orchestrator and Open Air Interface (OAI) for virtualizing the $\mathrm{CN}$. We demonstrate resiliency, and the effortless on-demand scaling or orchestrated auto-scaling using bin-packing or spreading strategies for achieving elasticity.
\end{abstract}

Index Terms-Docker Swarm, Microservices, Orchestration, Auto-scaling, Elasticity, Cloud Architecture.

\section{INTRODUCTION}

Fifth Generation $(5 \mathrm{G})$ of mobile network will serve different use cases with heterogeneous demands in terms of data rate, mobility, latency, reliability and energy efficiency. Reports forecast a continuation of the exponential increase of the number of smart-devices in addition to an anticipated 32 millions of Internet of Things (IoT) devices that will proliferate with 5G [1]. These devices will generate 44 Zettabytes of data per year [1]. Operators need to modernize their infrastructures to process this huge amount of data efficiently, with an adequate quality of experience at minimum spending to maximize their profitability. In this work, we are going to focus on the limiting factors on machines that are typically due to the finite and scarce nature of machine resources (Compute, Network and Storage) on infrastructure level. Resources provisioning is optimum when the "supply" meets exactly with the "demands". Prediction of required resource is used to prepare required resources ahead of time. However, on top of being computationally intensive and complex, predictions for scalability requires a sufficient amount of historical data to work properly, which is not always the case. In addition, sometimes, predictions are not even close to reality due to last minute changing circumstances or due to unforeseen factors. This results in losses due to over-provisioning. Elasticity in resources provisioning is a promising technology as it overcomes previous challenges on predictions' front. Network Function Virtualization (NFV) [2] is a network architecture that abstracts network nodes into building blocks as Virtualized Network Functions (VNFs) that will be deployed on a pool of resources enabling them to interconnect and chain easily to create communication services. Software Defined-Networking (SDN) [8] decouples the control plane from the switching plane making the network agile. Elasticity leverages NFV and SDN advents. It is well suited for cloud-based architecture using microservices [3]. On other hand, architects are changing the way they design their applications due to cloud emergence. Instead of monoliths, applications are decomposed into smaller and decentralized services so that they scale horizontally, by adding new instances as required. Leveraging cloud elasticity optimizes costs as it uses pay-as-you-go pricing [3]. Elastic resources are future-proof alternatives to predicted resources as they are reproducible and provisioned Just-inTime. To maintain affordability, elastic resources have to be orchestrated, using automated processes, to provision and de-provision resources as needed. This fits well in a cloud architecture using microservices. Microservices are simple, indivisible tasks that are easily instantiated, replicated, and scaled in a short period [3]. A group of microservices forms a holistic communication service and is considered as a "Slice of a network". In this demo, we will demonstrate orchestration of 4G/5G Core Network (CN) using NFV and SDN in a SoftwareDefined Data-Center (SDDC) context [9]. Our contribution is an NFV Orchestration (NFVO) Application (APP) enabling to

- create a cluster to host microservices

- auto-scale services using different orchestration strategies.

Note that we made publicly available the source code of our NFVO-APP at [10]. The proposed NFVO-APP provides needed services while optimizing network resource utilization. The remainder of this paper is organized as follows. In section II, we describe our platform architecture, while in section III; we provide an overview of our planned demonstration. 


\section{PlatForm Description}

We implement a virtualization of $4 \mathrm{G}$ LTE Evolved Packet Core (EPC) and 5G CN using "OpenAir Interface" (OAI-CN) [4] open-source software. We use Docker containers [5] to implement it as microservices. A container is a standard unit of software that packages up a code and all its dependencies so the application runs quickly, reliably on different computing environments and more additional security, as they are isolated in their contexts. Containers consumes fewer resources than Virtual Machines (VMs) as they share the same kernel with the host machine unlike VMs where the complete stack of a machine is implemented and thus consume additional memory. We orchestrate our system using Docker Swarm [5]. Swarm is a group of separate machines that will host our microservices. We implemented different strategies for provisioning microservices which are: "Bin-packing", and "Spread". "Bin-packing" assigns containers to one node until it is full before assigning them to another node. "Spread" assigns each container to the Swarm node with the most available resources, or in other terms, the least loaded node. The architecture of our platform is depicted in Fig. 1. It consists of provisioning several Swarm machines in order to secure High-Availability (HA). In our example, hierarchy is set to be one leader and five workers. For intercommunication, by default, we can rely on the internal routing mesh of the Docker Swarm manager to access a service on the target port of any node regardless of whether there is related microservice running on that node or not. Alternatively, we used an external load-balancer and reverse proxy for services with the internal routing mesh. Accordingly, HAProxy [6] or NGINX [7] is used to provide a floating IP address and caching service. In Fig. 1, service provisioning is done using "Spread" strategy to deploy LTE EPC implementation using OAI-CN [4]. This service is composed of several microservices. They are Mobility Management Entity (MME), Serving Gateway (SGw) and Packet Gateway (PGw) which are the building blocks of the EPC. On Swarm manager, we launch a script that runs indefinitely while monitoring sub-jacent services to control their size in term of Central Processing Unit (CPU) load as depicted in Fig. 2. It extracts CPU load on each service and according to predefined thresholds (threshold_l and threshold_h) takes a decision to scale-in/out (decrease/increase the number of microservices). A timeout phase follows any scaling in order to avoid snowball effect during execution.

\section{DEMO OVERVIEW}

Our architecture complies with the five pillars of software quality, that are scalability, availability, resiliency, management, and security [3]. The demonstration aims to illustrate this compliance. Using one script, we start by creating an HA Infrastructure composed of a Docker manager with 5 workers topped by the load-balancer/reverse proxy and the visualizer [10]. Next, we provision a CNaaS. We show the effect of different scheduling strategies on the provisioning of CNaaS while changing on-demand the scale of the OAI-

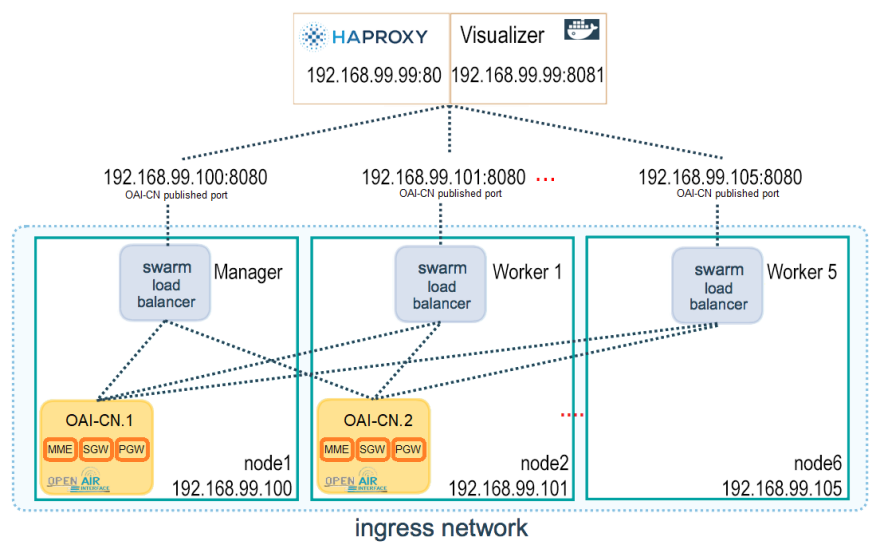

Fig. 1. OAI-CN (MME, SGW, PGW) provisioning with "Spread" Strategy

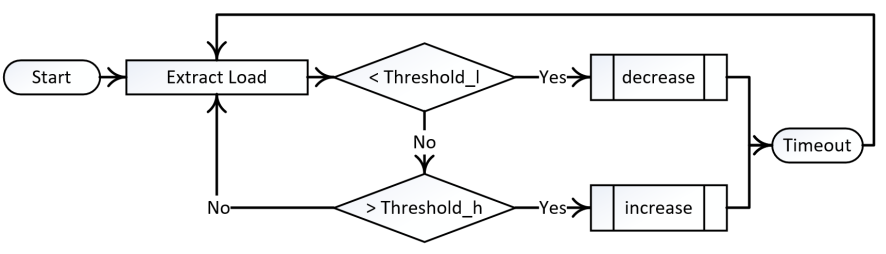

Fig. 2. Auto-Scaling Flowchart

CN VNF. This will illustrate the availability, scalability and ease of management. We demonstrate self-healing capability to show the resiliency in two scenarios. First consists of retuning the service during run-time forcing it to be transported to another Docker-machine of the Swarm cluster. Second is by killing the microservice itself. Both scenarios end up by autorecreation of the microservice. Third, we simulate an increase in load, so that we demonstrate the effortless auto-scaling using different orchestration strategies ("Binpacking" and "Spread") to show the auto-scaling of microservices without impacting other microservices due to isolation between Docker Swarm as security measures. 90 seconds demo video is available [11].

\section{ACKNOWLEDGEMENT}

This work was supported by the FUI SCORPION project (Grant no. 17/00464), "Azm \& Saade Foundation", and Lebanese University.

\section{REFERENCES}

[1] D Lund et al. "Worldwide and regional IoT-2020 forecast" AT\&T, 2014.

[2] Bo Han et al. "Network Functions Virtualization: Challenges and Opportunities" IEEE Comm. Surveys and Tutorials, 2014.

[3] Microsoft, Cloud Application Architecture Guide, Online: docs.microsoft.com/azure/architecture/guide/.

[4] Eurecom, "OpenAirInterface", Online: openairinterface.org/, Jul. 2015

[5] DOCKER, Enterprise Container Platform, Online: docker.com.

[6] HAProxy, Reliable TCP/HTTP Load Balancer, Online: haproxy.org

[7] NGINX, Load Balancer \& Reverse proxy, Online: nginx.org

[8] Open Networking Foundation, "TR-526 Applying SDN Architecture to 5G Slices", California, 2016.

[9] P. Marsh et al, 5G System Design - Architectural and Functional Considerations, Wiley, 2018

[10] Github, Online: github.com/nsalhab/docker-swarm

[11] Online: youtu.be/STZU_tV5ws8 